\section{AutoCPAP bei OSAS genauso effektiv wie Fixdruck-Variante}

Bloch KE et al. Autoadjusted versus fixed CPAP for obstructive sleep apnoea: a multicentre, randomised equivalence trial. Thorax 2018; 73: 174 - 184 doi:10.1136/thoraxjnl-2016-209699

Bei der obstruktiven Schlafapnoe (OSAS) verhindert die Beatmung mit konstant positivem Druck den Kollaps der Atemwege. Die Standardvariante mit einem fixierten Maskendruck verbesserte den Schlafkomfort, die Schlafeffizienz und Lebensqualität sowie hämodynamische $\mathrm{Pa}$ rameter. Die Studienresultate legen nahe, dass die Alternative mit automatisch adjustierten, bedarfsadaptierten Drücken ebenso wirksam ist.

Bei der Standardbehandlung mit Masken-CPAP erfolgt die manuelle Titrierung des später fixierten Druckniveaus (fCPAP) im Schlaflabor, damit in allen Schlafstadien und bei verschiedenen Körperpositionen eine Apnoe und Hypopnoe vermieden werden. Computer-kontrollierte Devices bieten ein kontinuierliches Feedback des Druckbedarfs mit einem Sensor und passen den Druck für den jeweils folgenden Atemzug an (auto(PAP). Daraus sollen ein durchschnittlich niedrigeres Druckniveau, geringere hämodynamische Effekte und eine bessere Akzeptanz vor allem bei Patienten mit periodischen, schweren Apnoen resultieren. Bloch et al. verglichen in einer multizentrischen, prospektiven Parallelgruppenstudie die langfristigen Effekte von fCPAP und autoCPAP. Zielvariablen waren u.a. die Tagesschläfrigkeit (Epworth Sleepiness Score ESS und OSLER-Test) und die Lebensqualität (SF-36, FOSQ und SF-6D). Nach 3, 12 und 24 Monaten erfolgten Schlafuntersuchungen mit Pulsoximetrie, Maskendruckbestimmung, Erfassung der Thoraxwandexkursionen und Körperpositionen sowie EKG. Weitere Parameter waren die 24-h-Blutdruckresultate, C-reaktives Protein und das Lipidprofil. Den Apnoe/HypopnoeIndex AHI und die Therapieadhärenz zuhause ergab die Auslesung der Geräte. Schließlich verglichen die Ärzte auch die Behandlungskosten.

Bei 208 Patienten (18-75 Jahre) mit unterschiedlich schweren OSAS erfolgte die Randomisierung. In allen Fällen bestand ein ESS $\geq 8$ und ein $A H I \geq 10 / h$. 80 (fCPAP) und 92 Patienten (autoCPAP) beendeten die Studie nach 24 Monaten. In beiden Gruppen verbesserten sich die objektiven und subjektiven Befunde (Intention-to-treat- und Per-Protokoll-Analyse). Insbesondere die Tagesmüdigkeit nahm ab. Die Effekte waren dabei vergleichbar (Intention-to-treat-Analyse: ESS fCPAP - 6,2 und autoCPAP - 6,3; OSLER-Test $+6,3$ min und $+8,3$ min). Verglichen mit den Ausgangsbefunden stieg die Lebensqualität signifikant. In beiden Gruppen normalisierten sich der $\mathrm{AHI}$ und der Oxygen-Desaturation-Index nach 24 Monaten. Die Wirkungen waren von einer Reduktion des systolischen, diastolischen und Mitteldrucks begleitet. Signifikante Gruppenunterschiede bestanden nicht. FCPAP und autoCPAP beeinflussten das C-reaktive Protein und das Lipidprofil nicht. Der mittlere Maskendruck war mit autoCPAP 2 mbar geringer als mit fCPAP. Die nächtliche Anwendungsdauer der Devices unterschied sich nicht (>5h/Nacht). Die Patienten bewerteten die Therapie als sehr effektiv. Unerwünschte Wirkungen (Maskenleck, Mundtrockenheit und nasale Obstruktion) waren in beiden Gruppen vergleichbar häufig.
FAZIT

Beide Device-Typen steigerten die Lebensqualität und verbesserten signifikant die objektiven Untersuchungsresultate. Die Autoren stufen fCPAP und autoCPAP als äquieffektive Optionen für die Erstlinientherapie des OSAS ein. Die Kostenanalyse ergab, dass OSAS-assoziierte Ausgaben einschließlich Fehlzeiten am Arbeitsplatz vor allem im ersten Jahr erfolgten. Auch die Unkosten wiesen keine Gruppenunterschiede auf.

Dr. med. Susanne Krome, Melle 